\title{
THE MIMAS-TETHYS AND ENCELADUS-DIONE SYSTEMS
}

\author{
A. VIENNE, L. DURIEZ AND S. CHAMPENOIS \\ Laboratoire d'Astronomie, Université de Lille 1
}

We have recently built a coherent theory of the motion of the satellites Mimas, Enceladus, Tethys, Dione, Rhea, Titan and Iapetus. The final form of the "Théorie Analytique des Satellites de Saturne" (TASS1.6) is presented in Vienne \& Duriez (1995). The internal precision of TASS is a few kilometers over three years and some tens kilometers over one century. The root-mean-square residuals of the adjustment of TASS over one century of Earth based observations reach $0^{\prime \prime} .12$ for the best data sets, until $0^{\prime \prime} .015$ for the few mutual phenomenas of 1981.

In the solution of Mimas and Tethys, nearly all arguments have been recognized as integer combinations of fundamental arguments which are :

$\phi_{1}$, so that $\phi_{1}-\left(\lambda_{o 1}-2 \lambda_{o 3}\right)$ is close to the proper pericenter of Mimas

$\phi_{3}$, so that $\phi_{3}-\left(\lambda_{o 1}-2 \lambda_{o 3}\right)$ is close to the proper pericenter of Tethys

$\Phi_{1}$, so that $\Phi_{1}-\left(\lambda_{o 1}-2 \lambda_{o 3}\right)$ is close to the proper node of Mimas

$\Phi_{3}$, so that $\Phi_{3}-\left(\lambda_{o 1}-2 \lambda_{o 3}\right)$ is close to the proper node of Tethys

$\omega_{1}$ is the libration argument of the resonance $\Phi_{1}+\Phi_{3}=0$.

where $\lambda_{o 1}$ and $\lambda_{o 3}$ are respectively the mean mean longitude of Mimas and Tethys. Among the arguments there are some very long periods :

$\alpha_{1}=\phi_{3}+2 \Phi_{1}$ (period: 200 years)

$\alpha_{2}=\phi_{1}-\phi_{3}+2 \Phi_{1}$ (period: 700 years)

In the model of Dermott et al. (1988) the semi major axis of the satellites grows up due to tidal dissipation on the planet. For example, Mimas and Tethys have crossed the resonance $2 \Phi_{1} 100$ million years ago (without locking inside it), and the system has evolved until to reach the present resonance $\Phi_{1}+\Phi_{3}$. Using the formulas of Dermott et al. and their value of the coefficient of the tidal dissipation, we have found that the commensurability $\dot{\alpha}_{1}=0$ has been reached 450000 years ago, and that $\dot{\alpha}_{2}=0$ will be reached in 190000 years. Of course, these "datations" are somewhat approximative but give an order of magnitude. In both cases, the semi-major axis are shifted of 1 to $3 \mathrm{~km}$ only, with $a_{1}=186009 \mathrm{~km}$ and $a_{3}=294958 \mathrm{~km}$. 


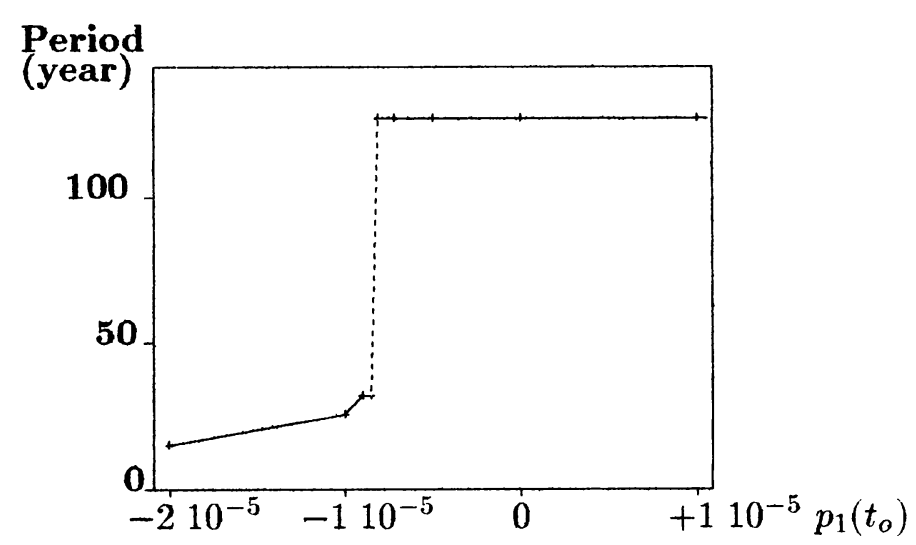

Figure 1. Variation of the frequency of $\alpha_{1}$ with respect to the initial contidion $p_{1}\left(t_{0}\right)$ (a variation of $p_{1}$ of $+110^{-5}$ corresponds to $-1,24$ kilometer in $a_{1}$ ).

We have studied the argument $\alpha_{1}$ only. It appears to be very sensitive to initial conditions. We have observed a skip in its frequency for a very small variation in the semi-major axis of Mimas (Fig 1). This skip is suspect. Furthermore, the frequency analysis failed for the integrations inside the skip (dot line).

As it was shown by Laskar $(1990,1992)$, the measure of the variation of the fundamental frequencies of a dynamical system can be related to the measure of chaos. We have computed the frequency $\dot{\omega}_{1}$ aver 200000 years with 130 evaluations of this frequency over 6000 years (intervals are overlaping at $75 \%$ ). This fundamental frequency shows significant variations $\left(10^{-5}\right.$ rad per year), but more computations are necessary to give a definitive conclusion. A similar study has shown that no significant variations exist for the fundamental frequencies of the Enceladus-Dione system.

These results are still preliminary. More computations and studies are necessary to confirm and understand a chaotic feature of the system MimasTethys. Neverthless, it is clear that these studies can not be neglected to understand the origin of the resonance under the effects of tital dissipation.

Dermott S.F., Malhotra R., Murray C.D.: 1988, Icarus 76, 295-334

Laskar J.: 1990, Icarus 88, 266-291

Laskar J., C. Froeschlé, A. Celletti: 1992, Physica D. 56, 253-269

Vienne A., Duriez L.: 1995, Astron. Astrophys. 297, 588-605 